REVUE DE L'INSTITUT

FRANÇAIS D'HISTOIRE

EN ALLEMAGNE
Revue de I'IFHA

Revue de l'Institut français d'histoire en Allemagne

$5 \mid 2013$

IFHA 5

\title{
Une vaste synthèse : l'Enzyklopädie der Neuzeit
}

\section{Guillaume Garner}

\section{OpenEdition}

\section{Journals}

Édition électronique

URL : http://journals.openedition.org/ifha/7387

DOI : 10.4000/ifha.7387

ISSN : 2198-8943

\section{Éditeur}

IFRA - Institut franco-allemand (sciences historiques et sociales)

\section{Édition imprimée}

Date de publication : 1 décembre 2013

ISSN : 2190-0078

Référence électronique

Guillaume Garner, «Une vaste synthèse : I'Enzyklopädie der Neuzeit », Revue de l'IFHA [En ligne], 5 | 2013, mis en ligne le 17 février 2014, consulté le 03 mai 2019. URL : http:// journals.openedition.org/ifha/7387 ; DOI : 10.4000/ifha.7387

Ce document a été généré automatiquement le 3 mai 2019.

(CIFHA 


\title{
Une vaste synthèse : l'Enzyklopädie der Neuzeit
}

\author{
Guillaume Garner
}

1 Seize volumes, 4000 articles, près de 10000 pages : l'Enzyklopädie der Neuzeit (EdN) tient d'emblée du monument et s'inscrit, déjà d'un point de vue quantitatif, dans la lignée de deux publications aînées et devenues des références incontournables auprès des historiens germanophones: Der Neue Pauly. Enzyklopädie der Antike et le Lexikon des Mittelalters. La prouesse que représente cette publication est d'autant plus remarquable qu'elle s'est faite en un temps très court : huit ans, à raison de deux volumes par an. Le maître d'œuvre de cette entreprise est l'historien Friedrich Jaeger, chercheur au Kulturwissenschaftliches Institut d'Essen, un Institut d'études avancées commun aux universités de Bochum, de Dortmund et de Duisbourg-Essen, spécialisé dans les sciences humaines et sociales.

2 Après avoir examiné les principes guidant l'organisation générale de l'EdN, on se demandera dans quelle mesure cette encyclopédie est un reflet de la pratique de l'histoire en Allemagne.

\section{Principes généraux}

3 1. S'adressant à la fois aux historiens universitaires ou professionnels et à un large public, l'EdN s'est fixée pour objectif de proposer un panorama encyclopédique de la Neuzeit, c'est-à-dire de la période allant de la fin du Moyen Âge au milieu du XIX ${ }^{\mathrm{e}}$ siècle (1848).

Cette vaste matière a été divisée en dix domaines d'études : État, interactions globales, droit et institutions, modes de vie et société, économie, sciences et médecine, formation, culture et communication, églises et cultures religieuses, littérature, arts et musique, environnement et évolution technique. Ces domaines ont été placés sous la responsabilité de coordinateurs (dont le nombre varie de deux à cinq), la supervision de thématiques plus spécifiques au sein de chacun de ces domaines étant assurée par un ou plusieurs 
responsable(s), spécialiste(s) de ladite thématique. Comme on le voit, l'architecture éditoriale est complexe, puisqu'elle associe 24 coordinateurs et 80 responsables. Ce point renforce le mérite des éditeurs qui sont parvenus à organiser le travail en commun d'une centaine des responsables à des degrés divers de cette entreprise.

Les articles se répartissent du point de vue de leur structure en trois catégories :

- une centaine d'«articles fondamentaux» (d'une dizaine de pages), parfois rédigés par plusieurs auteurs, propose une mise au point sur des phénomènes ou des évolutions caractérisant la période étudiée; citons à titre d'exemples les entrées "Renaissance ", « Réforme », « droit », « interactions globales » ou « travail ».

- un millier d'«articles généraux» (comprenant de trois à six pages) approfondit l'étude d'aspects centraux mentionnés dans les articles généraux ("vêtement», «climat», « chemin de fer », « révolte », « mesure du temps »)

- environ neuf cents articles particuliers (d'une à deux pages) sont consacrés à des thèmes précis (« amour parental », « jardin zoologique », « peur », « cantique », « maisonnée »).

Cette tripartition était destinée à éviter le risque d'un éclatement de la matière, alors que l'EdN est placée sous la bannière de la synthèse. Ce souci se traduit par le recours, classique dans une encyclopédie, à des renvois entre articles qui permettent d'approfondir l'étude d'une thématique précise. Il explique également que, d'emblée, la décision ait été prise d'écarter à la fois des articles biographiques et des articles traitant spécifiquement de l'histoire d'un pays. À propos de ce dernier point, il convient cependant de signaler que des phénomènes propres à un pays en particulier sont l'objet d'articles : c'est par exemple le cas pour la Bastille, le shogunat ou la common law.

6 L'approche retenue se veut, comme il est de mise dans une entreprise de synthèse de cette envergure, résolument pluridisciplinaire. La liste des dix domaines d'études retenus l'illustre, tout comme l'origine disciplinaire des responsables et coordinateurs de l'ouvrage qui balaie un large spectre correspondant à l'ensemble des sciences humaines et sociales. Les "historiens » sont évidemment majoritairement représentés, mais cette appellation est trop générale puisque l'EdN a fait appel à des spécialistes de disciplines historiques qui, à côté des chaires d'histoire générale, bénéficient d'un ancrage institutionnel ancien dans le paysage universitaire allemand et de chaires dédiées : c'est le cas de l'histoire des sciences, l'histoire du droit, l'histoire économique et sociale, l'histoire de l'Église (Kirchengeschichte), l'études des aires culturelles et/ou religieuses ( Afrikastudien, Asienstudien, Islamwissenschaft, etc.).

7 2. La périodisation choisie correspond également à cette recherche de synthèse globale qui caractérise l'élaboration de l'EdN. Ex negativo, cette période ne désigne pas la période moderne - la Frühe Neuzeit - mais bel et bien la Neuzeit. Le concept est difficilement traduisible en français et ne correspond pas aux découpages chronologiques qui structurent l'enseignement et la recherche historiques en France, puisque cette période englobe les quatre siècles qui séparent le milieu $d u X^{e}$ siècle du milieu du XIX ${ }^{e}$ siècle. Comme l'introduction de Fr. Jäger le précise explicitement, il s'agit d'intégrer à la fois la période moderne et la période révolutionnaire, la Revolutionäre Neuzeit, qui correspond au segment 1750-1850 et que Reinhart Koselleck a désignée sous l'expression - également intraduisible - de Sattelzeit (époque de transition).

Comment ce découpage chronologique est-il justifié ? Selon Fr. Jäger, considérer la Neuzeit comme une époque à part entière permet d'éviter l'approche consistant à voir dans la période moderne une sorte de période préliminaire des temps contemporains, un péché à laquelle une partie des recherches en Allemagne n'a pas toujours échappé. Mais il s'agit 
également de souligner les dynamiques et les évolutions qui font l'unité de cette période et de se démarquer ainsi d'une approche qui fait la part belle à la notion d'« Europe ancienne » (Alteuropa) et qui, notamment depuis les travaux d'Otto Brunner, souligne les éléments de continuité qui caractérisent l'Europe du XII ${ }^{\mathrm{e}}$ au XVIII ${ }^{\mathrm{e}}$ siècle, les années 1800 étant considérées comme la rupture majeure entre l'époque contemporaine (Moderne) et l'époque pré-contemporaine (Vormoderne).

Cependant, les éditeurs de l'EdN ne cachent pas que la date de 1450 s'est imposée avec plus d'évidence que la date terminale. Le choix de cette dernière est justifié par la double référence à la Sattelzeit de R. Koselleck et à la notion d'une période révolutionnaire caractérisant entre les années 1760 et 1848 l'Europe et le continent américain, ce qui revient à reprendre la thèse d'une révolution atlantique développée dans les années 1950 par J. Godechot et R. Palmer. À des évolutions profondes, surtout de nature économique et sociale, s'ajouterait donc la tentative d'établir un modèle encore largement pré-libéral de société civile, cette période d'expérimentations politiques se clôturant autour de 1848 . Alors que les raisons avancées pour justifier d'évolutions majeures dans la quasi-totalité des dix thèmes retenus autour de 1450 sont relativement plausibles (on y reviendra), l'argumentation en faveur de 1848-1850 semble moins assurée. Il s'agit surtout de se démarquer d'approches partant d'un long XIXe siècle, allant de 1789 à 1914 et qui a connu, quelques années après la publication des premiers volumes de l'EdN, une vogue après le succès spectaculaire de la monumentale somme de Jürgen Osterhammel consacrée à une histoire globale du XIX ${ }^{\mathrm{e}}$ siècle. Ce sont peut-être ces incertitudes qui expliquent des débordements hors cette date terminale, illustrés par exemple par un article sur le Kulturkampf. Il convient cependant de préciser que chez un spécialiste de l'historiographie aussi chevronné que Fr. Jäger, le fétichisme des dates et des périodes n'est pas de mise, leur choix répondant plus à des considérations heuristiques et interprétatives qu'à des fins de description de la réalité.

10 3. Le découpage spatial retenu découle de la définition d'une période en fonction d'évolutions et de processus avant tout propres à l'Europe - ce que les éditeurs assument explicitement. La dimension globale est présente dans l'EdN, mais elle est essentiellement abordée, dans les considérations introductives, à travers la problématique des interactions entre l'Europe et les autres continents, qui sont pensées en termes de relations d'influence réciproques. Certes, l'histoire européenne à la période étudiée ne peut plus se concevoir isolément de celle des autres continents, mais il n'en demeure pas moins que la globalisation était ici initialement envisagée à l'aune de l'Europe. De manière caractéristique, c'est sur ce point qu'ont porté les critiques lors de la session du dernier Historikertag consacrée à l'EdN. Ces critiques sont cependant à nuancer car, dans la pratique, ce programme d'une globalisation envisagée à l'aune de l'Europe a été parfois fortement nuancé (ce dont témoignent les réflexions conclusives du volume 16 sur les interactions globales) : si cette approche structure le long article consacré au thème (" Globale Interaktion»), d'autres articles en revanche abandonnent cette optique: celui consacré à l'Océan Indien propose ainsi un panorama complet des relations entre les aires et les espaces situés au bord et au sein de cet océan. En outre, de nombreux articles portent sur les aires et les espaces extra-européens, sans référence à l'Europe. C'est au total un certain pragmatisme qui l'a emporté, corrigeant ainsi une approche de la globalisation qui n'était pas immunisée contre le reproche d'eurocentrisme méthodologique. 
11 L'orientation principalement européenne de l'EdN interroge aussi la manière dont l'histoire européenne est envisagée, entre unité et hétérogénéité, en évitant à la fois la juxtaposition d'histoires nationales et la référence à un modèle dominant dont se distingueraient des voies particulières. En la matière, réaliser un parfait équilibre entre les différents espaces ou parties du continent européen aurait sans doute nécessité de réunir une équipe européenne d'historiens. Mais la plupart des auteurs étant allemands ou de langue allemande, un déséquilibre, inévitablement, s'introduit parfois au profit de l'espace allemand, puis des pays proches (îles britanniques, France, Italie, péninsule ibérique), aux dépens d'espaces plus lointains. Un exemple l'illustre: la guerre des Paysans (Bauernkrieg) est l'objet d'un article, la Grande Peur également, mais la révolte de Pougatchev n'a pas d'entrée spécifique.

12 Ce faisant, l'historien s'intéressant à l'histoire de l'Allemagne à l'époque moderne trouvera de nombreux articles sur ce domaine. On remarque en particulier le grand nombre d'articles consacré à l'Empire, et à ses institutions (diète, tribunal de la chambre impériale, Conseil impérial aulique), et la tonalité relativement consensuelle de ces articles, indiquant que les débats suscités par le livre de Georg Schmidt en 1999 sont terminés.

\section{L'entreprise dans le paysage historiographique : une pratique allemande de l'histoire?}

13 1. Comme on l'a évoqué, les auteurs sont majoritairement des universitaires ou des chercheurs exerçant en Allemagne ou dans des pays germanophones, les professeurs étant très largement représentés dans le groupe de la centaine de responsables et coordinateurs ayant supervisé l'EdN, les Privatdozente et enseignants-chercheurs postdoctorants étant minoritaires. En prenant la liste des responsables et coordinateurs (sachant que quelques-uns sont à la fois éditeurs d'un thème et coordinateurs d'un autre), on arrive à un total de 98 noms, dont 88 portent le titre de professeur (tous les professeurs n'étant pas titulaires d'une chaire ordinaire). Sur ces 98 noms, 92 exercent leurs fonctions en Allemagne, en Suisse ou en Autriche, trois en Angleterre, deux aux Pays-Bas et une à la fois en France et en Allemagne.

14 Ce point mérite d'être souligné car si, en Allemagne aussi le constat d'un éclatement ou d'un morcellement de l'histoire, a été dressé depuis trois décennies, ce morcellement y est compensé par la forte cohérence que donn à la profession d'historien un certain nombre d'institutions et de manifestations, dont les plus importantes sont l'association des historiens allemands et les congrès biennaux des historiens allemands (Historikertage). L'organisation d'une session de présentation et de discussion de l'EdN au cours du dernier Congrès des historiens allemands à Mayence en septembre 2012 était symptomatique du fort ancrage universitaire de cette dernière.

On peut ainsi penser que l'EdN a été d'emblée pourvue d'une légitimité à la fois institutionnelle et scientifique qui a contribué à son assise dans le champ de la recherche historique allemande, un coup d'œil sur la liste des auteurs montrant que les divers champs d'étude ont été confiés à des spécialistes reconnus de leur domaine. Ceci dit, relier la cohésion de l'EdN à des facteurs sociologiques et institutionnels serait réducteur : l'entreprise manifeste une véritable cohérence scientifique qui repose sur deux facteurs essentiels. 
16 2. Le premier de ces facteurs est la volonté affichée d'organiser l'ensemble des articles autour d'une interprétation globale de la période étudiée.

La lecture de l'introduction générale est emblématique de cette volonté de synthèse. L'unité et la signification de la période sont en effet justifiées par le recours à des hypothèses interprétatives et à des concepts qui montrent la portée qu'a, toujours aujourd'hui, la réception de la sociologie de Max Weber dans la pratique de l'histoire en Allemagne. Deux exemples illustrent ce point de vue. En premier lieu, parmi les dix arguments justifiant le choix du milieu du $\mathrm{XV}^{\mathrm{e}}$ siècle comme borne initiale de la Neuzeit, cinq au moins font référence aux thèses et aux notions de Max Weber : la formation de l'État moderne comme détenteur du monopole légitime de la violence, l'essor d'une économie capitaliste ou précapitaliste, l'affirmation d'une bourgeoisie qui remet progressivement en cause les fondements de la société d'ordres, la division confessionnelle consécutive à la Réforme protestante, et enfin l'affirmation d'un processus d'individualisation lié à l'essor de l'éducation et de l'alphabétisation. Le lecteur n'est par conséquent pas surpris de constater qu'une place importante est accordée à des concepts processuels (Prozessbegriffe) dont les historiens allemands sont friands: l'« individualisation ", la «disciplinarisation sociale » (Sozialdisziplinierung) sont ainsi l'objet d'un article spécifique.

18 En second lieu, on peut revenir sur la manière dont la périodisation est justifiée : conscients du caractère eurocentré du concept de Neuzeit tel qu'ils l'entendent, les éditeurs s'en justifient en citant les études d'histoire universelle de Max Weber selon lesquelles on ne peut, sans cette perspective européenne, appréhender les conditions de la formation du monde contemporain - tout en précisant que cette perspective doit être complétée et enrichie.

19 Une telle interprétation n'a cependant rien de doctrinaire. Il suffit pour s'en convaincre de lire conjointement l'introduction et les conclusions thématiques qui ouvrent le dernier volume de l'EdN. Il apparaît que cette interprétation générale est parfois confirmée, parfois nuancée, parfois réfutée par exemple en se référant aux nombreuses études micro-historiques ou d'histoire locale qui ont proposé un nouvel éclairage sur la formation de l'État moderne ou sur l'absolutisme, qui étaient traditionnellement présentés comme des processus imposés « d'en haut ».

20 Le second facteur de cohérence de l'EdN traduit les liens particuliers qu'entretiennent en Allemagne depuis le XIX ${ }^{\mathrm{e}}$ siècle l'histoire et la philosophie (deux disciplines qui étaient classiquement étudiées de concert). L'histoire de la philosophie occupe dans la liste des articles une place relativement importante qui peut surprendre un historien français. Mais ces liens étroits se traduisent surtout par une réception assez intense de l'histoire des concepts (Begriffsgeschichte) telle qu'elle a notamment été élaborée par Reinhart Koselleck dans les années 1970 et 1980. En témoigne l'ambition de l'EdN de combiner trois approches dans le choix et également dans le traitement des différents articles : la réalité historique et son évolution, l'historiographie et une histoire des concepts présents dans les sources. Disons-le franchement: la combinaison de ces trois niveaux est l'une des grandes qualités de l'EdN. De nombreux articles proposent ainsi une définition du thème étudié en combinant la prise en compte des sources et des évolutions historiographiques ; cette approche est particulièrement fructueuse car elle est appliquée à des termes présents dans les sources (etic) et dont on a parfois fait de manière non contrôlée des concepts interprétatifs (emic). L'article consacré à la notion de « subsistance » (Nahrung), qui a eu un impact considérable sur l'histoire économique et sociale en Allemagne en 
raison de l'utilisation qu'en a faite Werner Sombart dans son ouvrage sur le capitalisme, est représentative de cette approche. Autre exemple : l'article « Europe de l'Est » combine histoire du concept et présentation des grands traits de l'évolution de cette aire.

21 La combinaison de ces trois niveaux permet ainsi à l'EdN d'échapper aux impasses du positivisme plat et de la déconstruction sans fin, les travaux et études se rattachant au courant du tournant linguistique étant d'ailleurs dans l'ensemble assez peu mobilisés. Cette volonté de conjuguer ces trois niveaux (réalités, langue des sources, langue de l'historien) explique que les articles purement historiographiques soient rares (signalons par exemple l'entrée portant sur la "maisonnée » (ganze Haus) qui présente les débats ayant tourné autour de cette notion utilisée par Otto Brunner), mais de très nombreux «articles fondamentaux» et "articles généraux» proposent des mises au point historiographiques: on citera à titre d'exemples les articles sur la confessionnalisation, les corporations, la révolution agraire ou l'absolutisme.

3. La pratique de la pluridisciplinarité est une autre caractéristique de l'EdN, et elle se fait là encore selon des modalités spécifiques.

Une première caractéristique réside dans la part importante faite à l'histoire du droit dont témoignent les articles sur la police, l'administration ou les privilèges - et dans la participation d'historiens du droit renommés (entre autres Diethelm Klippel ou Heinz Mohnhaupt). On le sait : l'histoire générale allemande, parce qu'elle était avant tout une histoire politique centrée sur la formation de l'État, entretient par tradition des liens étroits avec l'histoire du droit. Cette dernière est de son côté dotée d'une forte identité disciplinaire, avec des chaires et des revues spécifiques, et elle s'est engagée depuis un demi-siècle dans une collaboration fructueuse avec l'histoire, dont l'un des acteurs majeurs est l'Institut Max-Planck d'histoire européenne du droit de Francfort. Depuis une vingtaine d'années, les liens ont concerné l'histoire du droit et l'histoire sociale autour de thématiques telles que la police, la criminalité ou l'histoire de l'administration locale. L'EdN traduit donc l'existence de liens entre historiens et historiens du droit qui sont bien plus resserrés qu'en France, d'autant plus que dans le sillage du courant des Annales, l'histoire moderne en France a longtemps accordé aux phénomènes juridiques une place très périphérique.

24 La pratique de la pluridisciplinarité dans l'EdN se traduit également par la présence de nombreux articles établissant une jonction entre histoire économique, histoire globale, histoire de l'environnement et histoire des techniques. La dimension économique de la « globalisation » est étudiée à travers des entrées telles que le commerce des Indes ou du Japon, un article sur la notion d'économie-monde ou économie mondiale (les deux termes correspondant au terme allemand de Weltwirtschaft). De très nombreux articles portent sur des matières premières ou des objets de la vie quotidienne (cuivre, zinc, savon, porcelaine, papier, etc.), et de nombreux autres combinent des approches d'histoire économique et d'histoire culturelle (articles "sucre", "café » entre autres) : l'article "thé» traite ainsi de l'économie mondiale du thé et de l'histoire culturelle de la consommation du thé. Ceci renvoie à une empreinte assez forte de l'anthropologie historique qui démontre, si besoin était, que les débats conflictuels entre histoire sociale et histoire du quotidien (Alltagsgeschichte) sont révolus.

Il s'agit bien de spécificités qui ne signifient évidemment pas que l'histoire en Allemagne soit un champ hermétiquement clos. L'EdN fournit ainsi des éléments permettant d'évaluer la réception des historiographies étrangères, moyennant des réserves qui 
tiennent au caractère forcément limité des compétences linguistiques des différents auteurs.

Les grands noms de l'historiographie italienne ou anglaise sont présents : Carlo Ginzburg est cité dans l'article sur «le sabbat des sorcières ", Giovanni Levi dans les entrées sur le village ou le marché foncier. De même, Lawrence Stone, Christopher Hill ou Edward P. Thompson (ce dernier entre autres dans les entrées «oisiveté ", «temps de travail», « industrie »). Le linguistic turn étant assez peu présent en Allemagne, les références à l'historiographie anglaise sont cependant parfois sélectives, comme en témoigne l'absence de Gareth Stedman Jones dans la bibliographie de l'article sur le chartisme. En ce qui concerne l'historiographie française, le courant des Annales est largement cité, en particulier dans les articles traitant de démographie historique, d'histoire des mentalités ou de structures sociales. Les références à Braudel sont également nombreuses et elles indiquent combien ce dernier est en Allemagne perçu comme l'auteur d'une histoire du capitalisme plus que comme celui de la Méditerranée.

Un pari très risqué et une réussite à la hauteur de l'importance du risque : tel est le sentiment qui prévaut à la consultation de cette encyclopédie qui a réussi à conjuguer les ambitions de la synthèse et du travail collectif. À en juger par les références en notes infrapaginales à l'EdN dans la production historiographique de ces toutes dernières années et par les comptes rendus (à la tonalité générale très positive) qui ont été consacrés aux différents volumes publiés, l'EdN se présente comme un monument fréquemment visité et qui est déjà en train de devenir incontournable dans le paysage historiographique allemand.

Lien internet : http://www.enzyklopaedie-der-neuzeit.de/

\section{AUTEUR}

GUILLAUME GARNER

ENS Lyon 